\title{
Electroluminescence of copper-nitride nanocrystals
}

\author{
Anna Stróżecka, ${ }^{1}$ Jingcheng Li, ${ }^{1,2}$ Robin Schürmann, ${ }^{1}$ Gunnar Schulze, ${ }^{1}$ Martina Corso, ${ }^{1,3,4}$ Fabian Schulz, ${ }^{1}$ Christian Lotze, ${ }^{1}$ \\ Sascha Sadewasser, ${ }^{5}$ Katharina J. Franke, ${ }^{1}$ and Jose Ignacio Pascual ${ }^{1,2,3}$ \\ ${ }^{1}$ Institut für Experimentalphysik, Freie Universität Berlin, Arnimallee 14, 14195 Berlin, Germany \\ ${ }^{2}$ CIC nanoGUNE, Tolosa Hiribidea 76, 20018 San Sebastian - Donostia, Spain \\ ${ }^{3}$ Ikerbasque, Basque Foundation for Science, Bilbao, Spain \\ ${ }^{4}$ Material Physics Center UPV-EHU, Manuel Lardizabal 5, 20018 Donostia - San Sebastián, Spain \\ ${ }^{5}$ International Iberian Nanotechnology Laboratory, Avenida Mestre José Veiga s/n, 4715-330 Braga, Portugal \\ (Received 19 December 2013; revised manuscript received 22 September 2014; published 17 November 2014)
}

\begin{abstract}
Nanocrystals can behave as quantum boxes with confined electronic states governing their optoelectronic properties. The formation of nanometer-size crystals of copper nitride $\left(\mathrm{Cu}_{3} \mathrm{~N}\right)$ grown by nitrogen sputtering of a $\mathrm{Cu}(110)$ surface is reported. Scanning tunneling spectroscopy shows that the nanocrystals exhibit a series of well-defined sharp electronic resonances, which correspond to confined free-electron-like states. We observe that electrons from a scanning tunneling microscope tip induce the emission of light with a larger efficiency than on the bare metal surface. The spectral analysis of the emitted photons reveals various radiative inelastic pathways enabled by the confined states, which explain the enhanced light emission. Thus, the $\mathrm{Cu}_{3} \mathrm{~N}$ nanocrystals can be employed as nanometer-size light sources.
\end{abstract}

DOI: 10.1103/PhysRevB.90.195420

PACS number(s): 78.67.Bf, 61.46.Km, 73.20.-r, 78.60.Fi

Semiconductor nanocrystals are potential sources for optoelectronic devices with size-dependent optical properties. Their coupling to light is enhanced by the proximity to high plasmonic fields such as those localized in rough surfaces or in metal nanoparticles [1,2]. Therefore, the interface with the metallic source of localized plasmons is critical because it needs to keep sufficient degree of electronic localization in the optically active nanocrystal and still allow electromagnetic coupling between metal and semiconductor nanostructures. Optimal integration of nanocrystals into optoelectronic devices would have a maximum coupling in contact to the metallic environment, which would also allow electrical currents to be used for excitation of optical emission. However, in this case, fast electron transitions between nanocrystal and metal environment are expected to lead to the quenching of the optical efficiency [3].

Here, we report the in situ growth of copper-nitride nanocrystals partly embedded in a copper surface, which show enhanced electroluminescence efficiency relative to its metallic environment. Bulk copper nitride $\left(\mathrm{Cu}_{3} \mathrm{~N}\right)$ is a semiconducting material, with an experimentally determined band gap ranging between 0.8 to $1.9 \mathrm{eV}$ [4]. One-atom-thick layers of copper nitride (i.e., $\mathrm{Cu}_{2} \mathrm{~N}$ ) can be grown by $\mathrm{N}^{+}$ sputtering on single-crystal $\mathrm{Cu}$ substrates [5-8]. The insulating properties of a single atomic layer of copper nitride have been used to decouple single atomic spins from an underlying metallic $\mathrm{Cu}(100)$ surface for scanning tunneling microscope (STM) characterization [9]. We find that the extended exposure of a $\mathrm{Cu}(110)$ surface to high energy $\mathrm{N}^{+}$ions leads to the formation of three-dimensional nanometer-size crystals of $\mathrm{Cu}_{3} \mathrm{~N}$, partially embedded into the surface of the copper substrate.

For the characterization of the nanocrystals' electro-optical properties we used scanning tunneling luminescence [10]. This technique allows direct comparison of optical spectra and quantum efficiencies with the electronic structure of the objects. Tunneling electrons through a quantum object can undergo radiative inelastic transitions between localized electronic states, mediated by the excitation of plasmon modes localized at the tunnel junction [10-12]. Tunneling electroluminescence has been applied to investigate electrooptical properties of nanostructures such as semiconducting quantum dots [13,14], metal nanoparticles [15], metallic quantum wells [16-18], or organic molecules [19-22].

We find that tunneling luminescence shows a larger quantum efficiency on the embedded copper-nitride nanocrystals than on the bare surface around them. The spectral analysis of the emitted light allows us to interpret this phenomenon as simultaneous radiative pathways enabled by the survival of localized resonances in the nano-object, proving in this way that sufficient degree of electron confinement persists in the semiconductor quantum box.

\section{EXPERIMENTAL DETAILS}

The experiments were performed in a custom-built lowtemperature $(5 \mathrm{~K})$ scanning tunneling microscope (STM), combined with dynamic atomic force microscopy (AFM) using a qPlus sensor [23], operating in ultrahigh vacuum. Light emitted from the STM junction was collected and focused into the entrance slit of a grid spectrometer (Acton Research Corporation SP150) coupled to a liquid-nitrogencooled silicon charge-coupled device (CCD) camera (Spec-10, Princeton Instruments). The detected spectral range is $400 \mathrm{~nm}$ to $1000 \mathrm{~nm}$.

We estimate the total quantum efficiency from the integral of the light emission spectra normalized by the electron current, $I_{t}$, and by the acquisition time, $t$. The resulting value is multiplied by a factor of 30 , accounting for the small solid angle of our detection setup. This value refers only to the narrow spectral window of our detector.

$\mathrm{Cu}_{3} \mathrm{~N}$ nanocrystals were grown by exposing an atomically clean $\mathrm{Cu}(110)$ substrate to a $600 \mathrm{eV} \mathrm{N}^{+}$ion sputtering current $(I=10 \mu \mathrm{A})$ for three minutes, with $1 \times 10^{-4} \mathrm{~N}_{2}$ partial pressure, $700 \mathrm{eV}$ beam energy, and sputtering current exceeding $I=10 \mu \mathrm{A}$. The sample temperature was kept at 


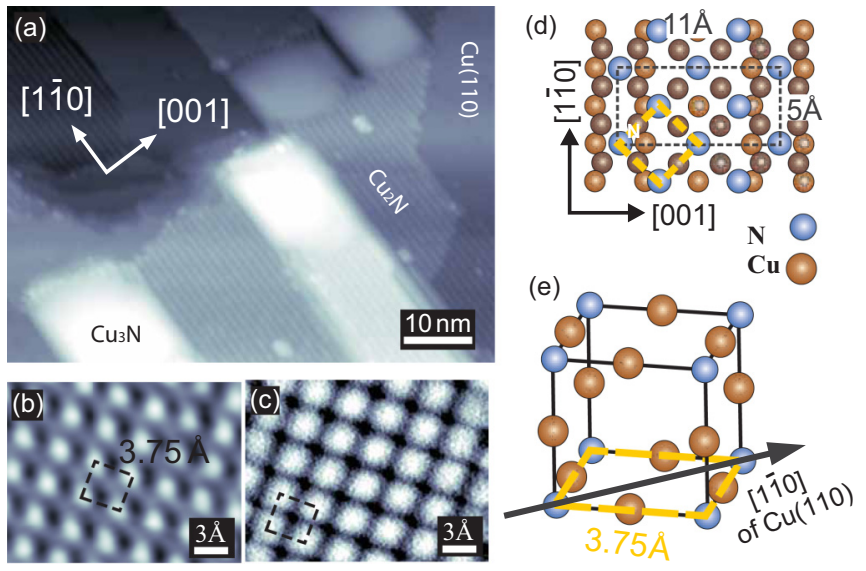

FIG. 1. (Color online) (a) STM image of copper-nitride nanocrystals formed on $\mathrm{Cu}(110)$. The corrugated striped phase corresponds to the $\mathrm{Cu}_{2} \mathrm{~N}$ monolayer, and the bright rectangular protrusions are the $\mathrm{Cu} 3 \mathrm{~N}$ nanocrystals. (b), (c) Atomically resolved structure of $\mathrm{Cu}_{3} \mathrm{~N}$ nanocrystals: tunneling current STM image (b) and AFM (c). The size of the unit cell obtained from the images fits with the unit cell dimensions of the (100) face of a $\mathrm{Cu}_{3} \mathrm{~N}$ crystal. (d) Schematic structure of $\mathrm{Cu}_{3} \mathrm{~N}$ monolayer. The $\mathrm{N}$ atoms are incorporated into the $\mathrm{Cu}$ layer. The gray rectangle marks the unit cell of the monolayer, the yellow square the unit cell of the nanocrystal. (e) Schematic crystal structure of three-dimensional $\mathrm{Cu}_{3} \mathrm{~N}$. The arrow shows the direction of stripes in the $\mathrm{Cu}_{3} \mathrm{~N}$ network (image processing using WSxM [36]).

$700 \mathrm{~K}$. After further annealing at this temperature for two minutes the sample was cooled down and inserted into the low-temperature STM.

\section{RESULTS AND DISCUSSION}

Figure 1(a) shows an STM overview of the $\mathrm{Cu}(110)$ surface upon nitriding. The surface appears mostly covered by a copper-nitride monolayer $\left(\mathrm{Cu}_{2} \mathrm{~N}\right)$, exhibiting a characteristic striped superstructure due to the different matching periodicity of layer and surface [Fig. 1(d)] [24]. In addition, rectangular objects with apparent height of a few angstroms and lateral sizes between 8 and $30 \mathrm{~nm}$ appear embedded in the $\mathrm{Cu}_{2} \mathrm{~N}$ network [Fig. 1(a)]. These objects may appear pinning step bunches, and sometimes even buried under the surface [see Fig. 2(a)]. They also have a different electronic structure than the surrounding $\mathrm{Cu}_{2} \mathrm{~N}$ (see below). These facts suggest that these objects have a three-dimensional structure, which is embedded within the copper surface.

\section{A. Structure of the nanocrystals}

To find out the nature of these objects, we elucidated their structure using combined STM and AFM measurements. Atomically resolved images of the surface of these objects [Figs. 1(b) and 1(c)] show that their topmost facet exhibits a square lattice with unit cell vector of $3.75 \AA$, fitting with the atomic structure of the (100) surface of a $\mathrm{Cu}_{3} \mathrm{~N}$ crystal [shown sche-matically in Fig. 1(e)] [25]. This is the same atomic structure as $\mathrm{Cu}_{2} \mathrm{~N}$ layers grown on the $\mathrm{Cu}(100)$ surface, and also is close to the structure of the nitride layer grown on the bare $\mathrm{Cu}(110)$ surface, however without the characteristic ripple (Fig. 1). Hence, we identify them as three-dimensional nanocrystals of copper nitride $\left(\mathrm{Cu}_{3} \mathrm{~N}\right)$ [25].

The 3D growth of $\mathrm{Cu}_{3} \mathrm{~N}$ is kinetically facilitated at elevated temperatures, when the energy and the flux of $\mathrm{N}^{+}$ ions are sufficiently large to allow crystal nucleation into the $\mathrm{Cu}$ bulk [8]. The density of $\mathrm{Cu}_{3} \mathrm{~N}$ nanocrystals on the $\mathrm{Cu}_{2} \mathrm{~N} / \mathrm{Cu}(110)$ surface can be tuned by varying the growth parameters such as $\mathrm{N}^{+}$ion sputtering current, sputtering time, or substrate temperature.

The nanocrystals are partly embedded inside the copper bulk and protrude for several angstroms above the sample [Fig. 2(a)]. The exposed surface of the nanocrystals generally presents a curved-like topographic shape, as resolved by STM and AFM profiles [Fig. 2(c)]. Furthermore, no sharp boundaries are found between the nanocrystals and the $\mathrm{Cu}_{2} \mathrm{~N}$ network along the $[1,-1,0]$ direction. Instead, in some cases the $\mathrm{Cu}_{2} \mathrm{~N}$ film apparently evolves continuously at the boundary between the $\mathrm{Cu}_{2} \mathrm{~N}$ on $\mathrm{Cu}(110)$ and the nanocrystal [Fig. 2(b)], suggesting that the topmost layer of both areas is commensurate in a continuous nitride film covering the nanocrystal. In fact, $\mathrm{Cu}_{2} \mathrm{~N}$ monolayers on $\mathrm{Cu}(110)$ have been proposed to be a precursor state for $\mathrm{Cu}_{3} \mathrm{~N}$ crystal growth on $\mathrm{Cu}(110)$ surfaces [26].

Summarizing the structural analysis of these nano-objects, we identify them as three-dimensional nanoclusters of copper nitride partially buried in the copper surface with their (100) facet close to perpendicular to the surface and covered by a extended atomic-thick nitride layer.

\section{B. Electronic configuration}

An interesting aspect of these semiconductor nanocrystals is that they enclose a set of discrete unoccupied electronic levels, which potentially makes these nanoparticles interesting for optical applications. First, in Fig. 3, we compare $d I / d V$ spectra measured on a nanocrystal and on the bare $\mathrm{Cu}_{2} \mathrm{~N}$ layer. The spectrum on the layer exhibits two broad resonances $\left(\mathrm{S}_{1}\right.$ and $\mathrm{S}_{2}$ ) at about $1.8 \mathrm{eV}$ and $3.8 \mathrm{eV}$, which were previously attributed to free-electron-like surface states of the nitride layer [27,28]. On the $\mathrm{Cu}_{3} \mathrm{~N}$ nanocrystals, the $d I / d V_{s}$ spectra show similar resonances $S_{1}$ and $S_{2}$ as those found on the $\mathrm{Cu}_{2} \mathrm{~N}$ layer, but much shaper, slightly shifted, and each of them accompanied by a characteristic substructure, labeled as $S_{1}^{\prime}$ and $\mathrm{S}_{2}^{\prime}$.

Wider range spectra such as the one in Fig. 4 shows that the nanocrystals also exhibit a lower-lying resonance, labeled QW in the spectrum shown in Fig. 4. The energy position of the QW state is here $0.05 \mathrm{eV}$ above $E_{f}$, but it varies in the range between -0.1 and $0.8 \mathrm{eV}$ depending on the crystal. We found no correlation of the QW alignment with the width of the crystal. Therefore, we interpret it as a state vertically confined within the nanocrystal, with its energy position depending on the vertical dimension of the embedded crystal.

Interestingly, the QW state appears with a characteristic substructure in its high-energy tail. To identify its origin, we set the applied bias at various energies along the resonance's tail and measure spatial maps of the $d I / d V_{s}$ signal, shown in Fig. 5(a) for a nanocrystal with a $\mathrm{QW}$ at $0.45 \mathrm{eV}$. At the QW onset, the $d I / d V_{s}$ maps show a single lobe at the center, but at 

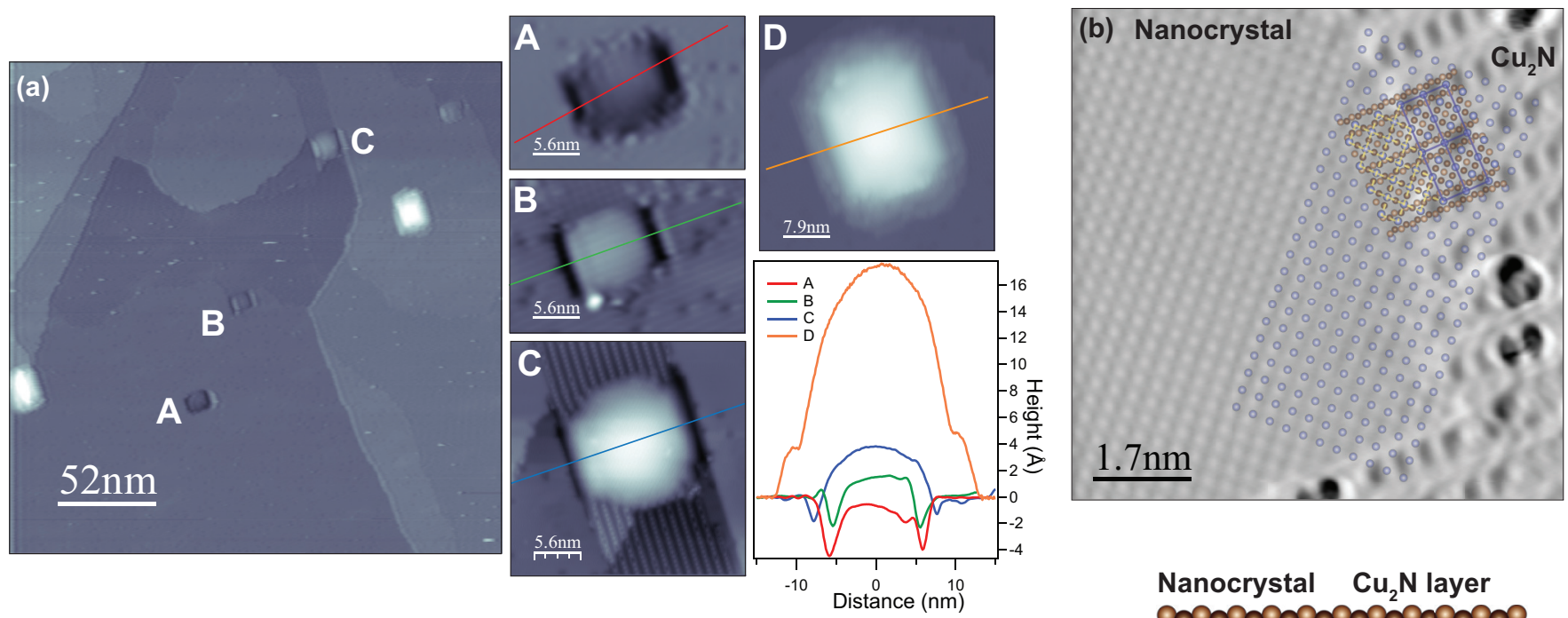

(c)
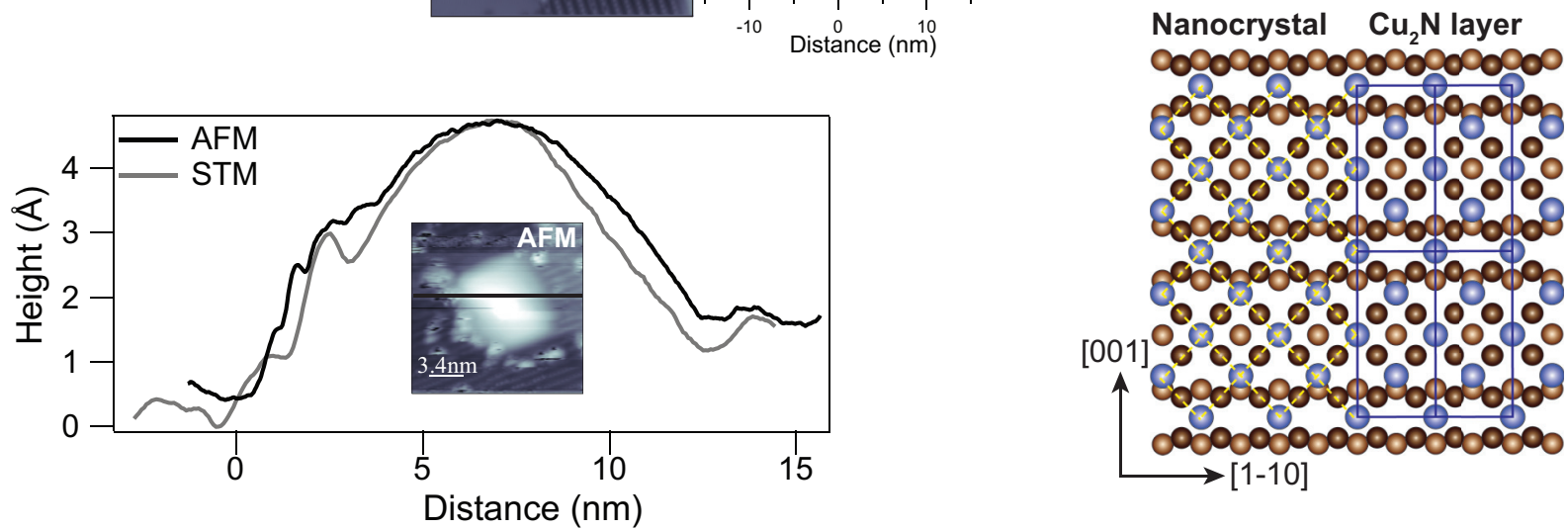

FIG. 2. (Color online) (a) Large-scale STM image of a $\mathrm{Cu}(110)$ surface partially covered by the $\mathrm{Cu}_{2} \mathrm{~N}$ network, showing different types of $\mathrm{Cu}_{3} \mathrm{~N}$ nanocrystals. (A), (B) Nanocrystals embedded within the $\mathrm{Cu}$ surface, (C) nanocrystal grown on $\mathrm{Cu}_{2} \mathrm{~N}$ terraces, (D) nanocrystal protruding above clean $\mathrm{Cu}$ areas. Height profiles taken along the plotted lines above the different nanocrystals are shown in the graph. (b) STM-current (error signal) image of a region at the border between a nanocrystal and the $\mathrm{Cu}_{2} \mathrm{~N}$ film. The atomic lattice is continuous as shown in the model. (c) Height profiles of a nanocrystal (inset) show a curved shape in both STM and AFM measurements. Electronic effects in the measurement are excluded since AFM data are taken in $\Delta f$ feedback mode.

100 meV higher energy a substantially different feature with a nodal plane appears. These patterns are characteristic of lateral electron confinement. Hence, we attribute the substructure above the QW onset to the discrimination of this state due to the finite lateral sizes of the crystal.

Maps of the $d I / d V$ amplitude of resonances $\mathrm{S}_{1}$ and $\mathrm{S}_{2}$ reveal that each of the two states appearing as a substructure

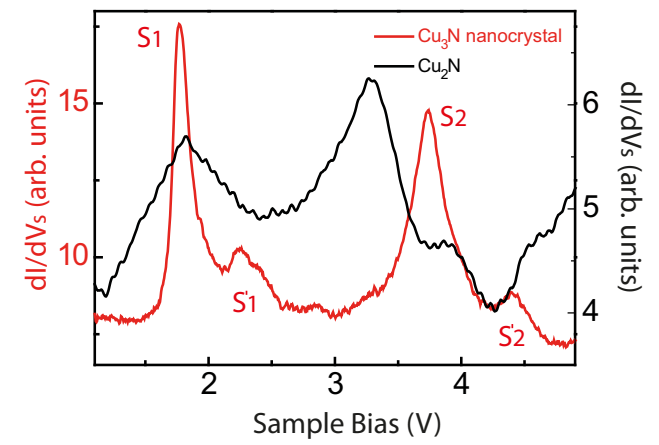

FIG. 3. (Color online) Comparison of constant-current $d I / d V$ spectra recorded on $\mathrm{Cu}_{3} \mathrm{~N}$ nanocrystal and on the bare $\mathrm{Cu}_{2} \mathrm{~N}$ covered $\mathrm{Cu}(110)$ surface.
[ $S_{1}-S_{1}^{\prime}$ and $S_{2}-S_{2}^{\prime}$ in Fig. 3] is imaged with different spatial extensions within the nanocrystal [Fig. 5(b)]. The lower of each doublet covers the whole area of the crystal, whereas the

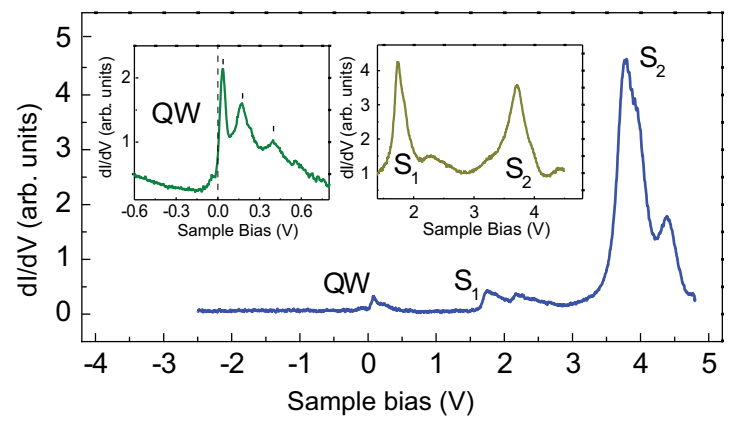

FIG. 4. (Color online) $d I / d V_{s}$ spectrum recorded on a $\mathrm{Cu}_{3} \mathrm{~N}$ nanocrystal. The tip-sample distance was linearly decreased with bias $(2.8 \AA)$ in order to extend the dynamic range of the current voltage converter to cover the wide range of bias values. Various features are labeled as QW (quantum well state), $\mathrm{S}_{1}$, and $\mathrm{S}_{2}$ (surface states), and described in the text. The insets show constant-height (left) and constant-current (right) $d I / d V_{s}$ spectra around the energy of the resonances $\mathrm{QW}$, and $\mathrm{S}_{1}$, and $\mathrm{S}_{2}$, respectively. 
(a)
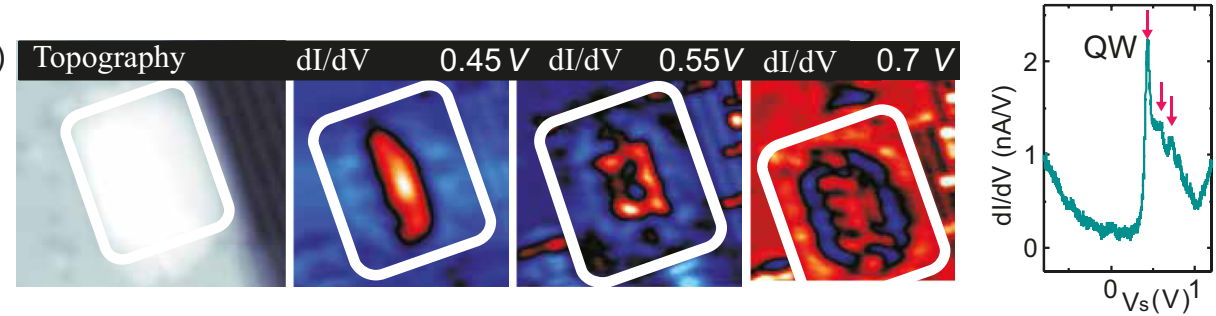

(b)
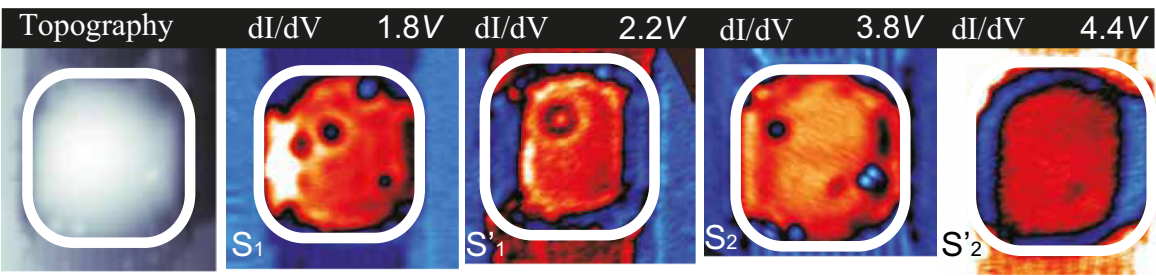

FIG. 5. (Color online) (a) QW state: $d I / d V$ images at the bias corresponding to the substructure marked with arrows in the spectrum shown in the rightmost panel. (b) Similar $d I / d V_{s}$ maps of resonances $\mathrm{S}_{1}$ and $\mathrm{S}_{1}^{\prime}$ and $\mathrm{S}_{2}$ and $\mathrm{S}_{2}^{\prime}$ for a different nanocrystal.

higher one appears localized in a smaller region. We suspect that this specific difference in the extension might reflect that the lower of each state $\left(S_{1}\right.$ and $\left.S_{2}\right)$ corresponds to the $\mathrm{Cu}_{2} \mathrm{~N}$ overlayer state covering the crystal, and the higher one $\left(S_{1}^{\prime}\right.$ and $S_{2}^{\prime}$ ) to an interface state of the crystal underneath. The sharper line shape of the $S_{1}$ and $S_{2}$ states with respect to those equivalent on the bare nitride layer (Fig. 3) can be interpreted as a larger electronic decoupling due to the presence of a nitride nanocrystal underneath.

\section{Electroluminescence of the nanocrystals}

The set of discrete electronic resonances in the embedded copper-nitride nanocrystals confers on them interesting electroluminescent properties. We have detected the light emitted from the nanocrystals during electron tunneling using the optical setup coupled to our low-temperature STM. Figure 6(a) compares a light emission (LE) spectrum on the $\mathrm{Cu}_{3} \mathrm{~N}$ nanocrystal presented in Fig. 3 with two reference spectra obtained with the same STM tip on nearby $\mathrm{Cu}(110)$ and $\mathrm{Cu}_{2} \mathrm{~N}$ areas, respectively. For an applied bias of $1.6 \mathrm{~V}$ we observe a sharp cutoff at $h v=1.6 \mathrm{eV}$, above which no photons are detected due to energy conservation. The intensity of the light emitted from the nanocrystal per tunneling electron, $I(h v)$, is larger than from either the bare surface or the $\mathrm{Cu}_{2} \mathrm{~N}$ layer. The quantum efficiencies obtained from Fig. 6(a) are $1.6 \times 10^{-6}$, $1.0 \times 10^{-6}$, and $0.8 \times 10^{-6}$ for the $\mathrm{Cu}_{3} \mathrm{~N}$ nanocrystal, $\mathrm{Cu}_{2} \mathrm{~N}$ layer, and $\mathrm{Cu}(110)$, respectively. These results show that for this applied bias, light emission from the nanocrystal is about 1.6 times more efficient than from the $\mathrm{Cu}_{2} \mathrm{~N}$ layer and has twice the efficiency of light from the bare copper surface.

The LE spectra show marked features associated with plasmon modes localized at the tunneling junction [4]. These cavity plasmon modes depend on details of the tip-apex structure and, therefore, change as the tip is modified. To extract quantitative information from the spectra, we normalized all photon intensity spectra $I(h v)$ by a reference spectrum $I_{\text {ref }}(h v)$ measured for each given tip on the bare surface and with
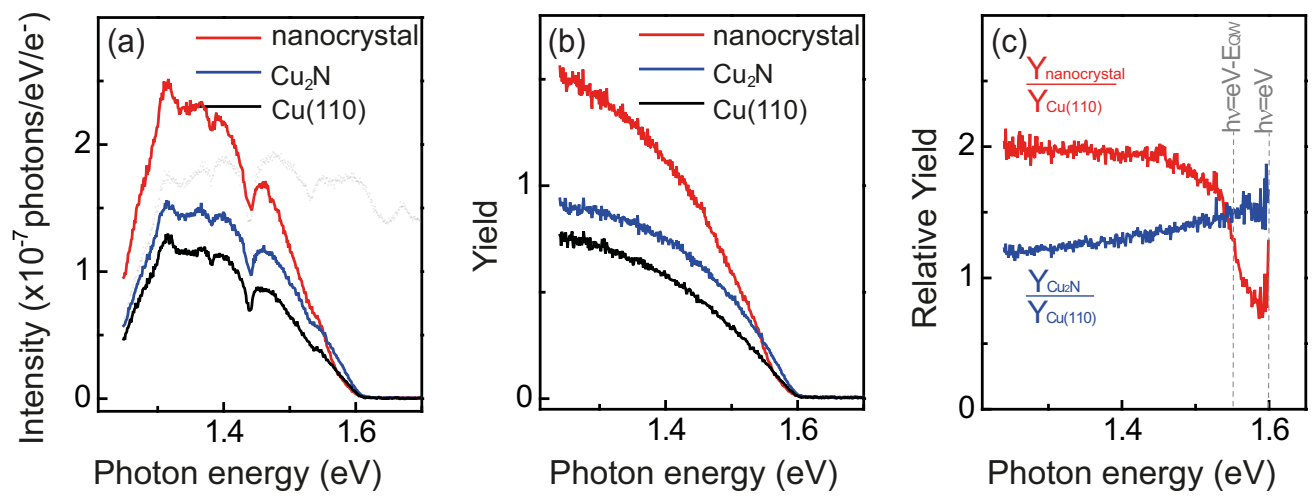

FIG. 6. (Color online) (a) Comparison of light intensity per tunneling electron $I(h v)$ spectra obtained on the $\mathrm{Cu}_{3} \mathrm{~N}$ nanocrystal of Fig. 2 , on the $\mathrm{Cu}_{2} \mathrm{~N}$ layer, and on the $\mathrm{Cu}(110)$ surface $\left(I=20 \mathrm{nA}, V_{s}=1.6 \mathrm{~V}\right.$, time $\left.=180 \mathrm{~s}\right)$. The dotted gray line shows the reference spectrum, measured on a bare $\mathrm{Cu}(110)$ region with $V_{s}=3 \mathrm{~V}$, used to normalize the data in (b). (b) Plots of the spectral yield $Y(h v)$ obtained by normalization of the data of (a). By removal of the plasmon-related features, the resulting spectra are tip-independent, as described in the Appendix. (c) Spectral yields of a $\mathrm{Cu}_{3} \mathrm{~N}$ nanocrystal and of the $\mathrm{Cu}_{2} \mathrm{~N}$ layer relative to that of the $\mathrm{Cu}(110)$ surface. The plots quantify the larger photon emission efficiency of the nanocrystal, and reveal a photon energy cutoff associated to the position of the QW resonance. 
applied bias above the spectral range of the photon detection (e.g., $3 \mathrm{eV}$; see the Appendix). The resulting spectral yield function, defined as $Y(h v)=I(h v) / I_{\text {ref }}(h v)$, can be used to compare spectra acquired with different currents and is not sensitive to the tip structure.

The spectral yield of the data in Fig. 6(b) shows now smoothly decreasing functions going to zero at the photon energy corresponding to the applied bias $\left(V_{s}=1.6 \mathrm{~V}\right)$. On the nanocrystal, the photon yield is larger, but the spectrum has a different cutoff energy. These effects are more clearly shown plotting the relative yields using the spectrum on the bare copper as a reference [Fig. 6(c)]. Photon emission on the $\mathrm{Cu}_{3} \mathrm{~N}$ nanocrystal has double efficiency of the $\mathrm{Cu}(110)$ surface in most of the spectral window, but decreases to a similar value at about $0.05 \mathrm{eV}$ before the cutoff set by the bias voltage $(1.6 \mathrm{eV})$. We note that this particular crystal has a QW state at $0.05 \mathrm{eV}$ above $E_{f}$. The light onset at $h v=1.55$ $\mathrm{eV}$ is then interpreted as due to inelastic tunneling into this localized state as final state. This radiative transition emits light in parallel to transitions into $E_{f}$, the radiative channel active all over the $\mathrm{Cu}_{2} \mathrm{~N}$ layer, thus explaining the larger LE efficiency of the nanocrystal.

The evolution of LE yield spectra with the applied bias reveals additional radiative transitions within the nanocrystal. Figure 7(a) shows a color map of spectral yield vs applied

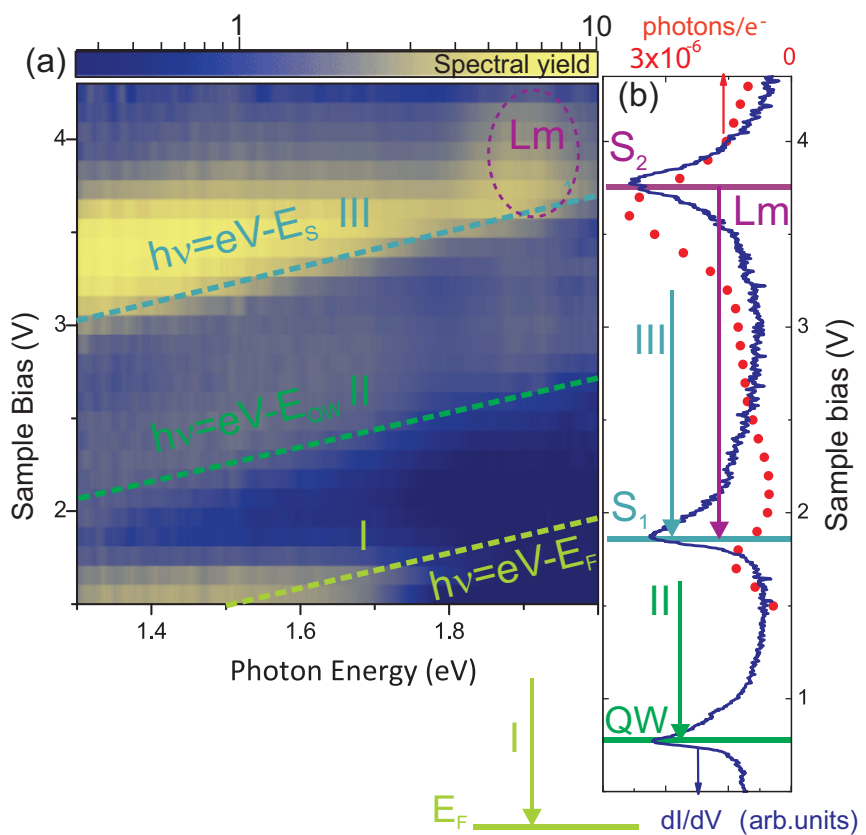

FIG. 7. (Color online) (a) Photon yield spectra versus applied bias. The photon yield (color coded) is obtained using a reference spectrum measured on the bare $\mathrm{Cu}(110)$ surface at $V_{s}=3 \mathrm{~V}$. Dashed lines mark cutoffs in the spectra which shift linearly with the applied bias. These correspond to the maximum photon energy for a certain transition into a resonance, and thus allow identifying the different final states. The different radiative transitions observed are labeled as I, II, III, and Lm. Each spectrum is acquired for $120 \mathrm{~s}$, with $I_{t}=50 \mathrm{nA}$. (b) $d I / d V_{s}$ (constant-current mode, $I=0.5 \mathrm{nA}$ ) acquired over the nanocrystal with a QW state at $0.8 \mathrm{~V}$. Arrows indicate the decay processes identified in (a). Dotted red curve shows the integrated photon counts. voltage for a nanocrystal with a $\mathrm{QW}$ state at $0.8 \mathrm{eV}$. The corresponding $d I / d V_{s}$ spectrum of the nanocrystal is shown in Fig. 7(b). The spectral map shows several onsets of emission at photon energies that evolve linearly with the applied bias (dashed lines I, II, and III). These onsets indicate the maximum photon energy for a given transition, i.e., $h v=e V_{s}-E_{\text {final }}$, and therefore, they are a fingerprint of the electronic final state driving the photon emission [shown with arrows in Fig. 7(b)]. At the energy of the $S_{1}$ resonance, radiative transitions into $E_{f}(\mathrm{I})$ vanish. Above it, light from transitions into the $\mathrm{QW}$ resonance (II, onset $h v=e V_{s}-E_{\mathrm{QW}}$ ) appear still in the spectra, but with less intensity. A new transition into the $S_{1}$ resonance (III) appears as the photon energy $h v \leqslant e V_{s}-E_{S_{1}}$ reaches the spectral window of our setup, and also decreases in intensity at the bias of resonance $S_{2}$. At this point, a peak at a fixed photon energy $\sim 1.9 \mathrm{eV}$ (labeled Lm) develops over an otherwise featureless yield spectrum. Contrary to the voltage dependence of photon yield onsets I, II, and III, the position of the Lm peak is independent of the applied sample bias [also shown in Fig. 8(a)]. This suggests that this emission process arises from transitions between two resonances in the nanocrystal, i.e., a luminescence-like process [16]. In fact, the peak's energy agrees with the energy difference between resonances $S_{2}$ and $S_{1}$, which would be then initial and final states in the radiative electron transition [16].

An interesting outcome of the spectral map is that it allows us to compare the quantum efficiency (QE) of the intraluminescence process Lm with that of channels I-III. The fraction of light emitted by the Lm luminescence channel is estimated from the ratio of areas of the $\mathrm{Lm}$ peak and background, using (plasmon-normalized) yield spectra like in Fig. 8(a) [29]. This allows us to obtain the relative strength of the Lm channel over the total light emission, independent of the specific plasmon structure of the tip.

Figure 8(b) plots the evolution of the QE with the tunneling bias for both the total light emission (in the spectral range of our detector) and the Lm emission channel. The total QE increases until $V_{s} \sim 3.6 \mathrm{eV}$, when the applied bias reaches the value of resonance $S_{2}$. From the map in Fig. 7, we identify these photons as coming from direct electron decay into resonance $\mathrm{S}_{1}$ (transition III). As soon as resonant tunneling through $\mathrm{S}_{2}$ becomes active, the light emission from this radiative channel decreases [30]. The $S_{2} \rightarrow S_{1}$ transitions (Lm) can then be detected, with $\mathrm{QE}$ peaked at the value of resonance $\mathrm{S}_{2}$, and leveling off above it to a roughly constant plateau. This emission channel is only active to electrons tunneling resonantly through the $S_{2}$ state, but its efficiency is small (a factor of 15 smaller than the total QE). This fact indicates that resonant tunneling through localized states in the nanocrystals has a weaker radiative (inelastic) cross section. For energies above the $S_{2}$ state, the Lm emission will occur after the tunneling electron has undergone a low-energy transition from its initial state to the intermediate state $S_{2}$ [process IV in Fig. 8(c)]. The radiative efficiency of channel IV is presumably small because of the lower plasmon enhancement in the mid-infrared range (and in any case, could not be detected, because it lies outside the spectral window of our detector). Therefore, the efficiency of the Lm process decreases gradually with applied bias. 

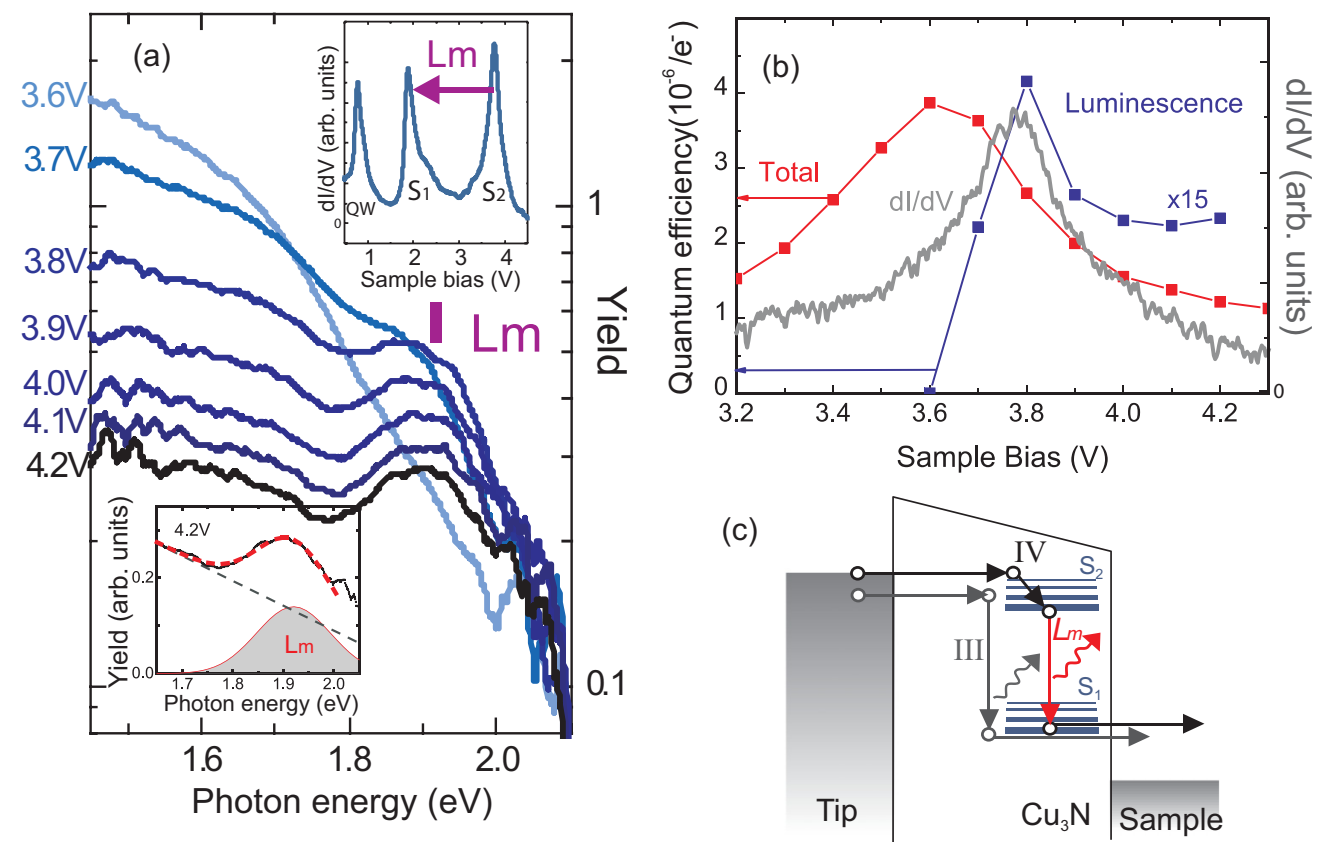

FIG. 8. (Color online) (a) Evolution of the photon yield spectra $Y(h v)$ from Fig. 7 at sample bias between 3.6 and $4.3 \mathrm{~V}$. The peak Lm which evolves in the yield spectra corresponds to the direct electronic transition between the states $S_{2}$ and $S_{1}$ of the nanocrystal, as shown in the top right inset image. The bottom left inset shows the fit of the luminescence spectra by a Gaussian peak. (b) Bias dependence of the total quantum efficiency and the quantum efficiency of the luminescence process. (c) Schematic image of the inelastic decay processes giving rise to direct (III) and internal (Lm) radiative transition mechanisms.

\section{CONCLUSIONS}

We have shown that $\mathrm{Cu}_{3} \mathrm{~N}$ nanocrystals appear embedded into the surface of a $\mathrm{Cu}(110)$ substrate after extensive dosing of $\mathrm{N}^{+}$ions. The crystals behave as quantum electron boxes with enhanced efficiency for the emission of light from tunneling electrons. The spectral analysis of the emitted light revealed that multiple electroluminescence channels operate in parallel, explaining the larger efficiency. Most of the light comes from direct inelastic transitions of tunneling electrons, decaying into nanocrystal states. Electrons injected directly at resonances show a weaker radiative behavior, but still produce detectable intracrystal luminescence transitions. These results demonstrate that various radiative decay channels act additively in the conversion of electrical to optical energy, and in this way may improve the photon emission efficiency of quantum electron boxes.

\section{ACKNOWLEDGMENTS}

The research is supported by the DFG, via priority program SPP 1243, STR 1151/1, and SFB 658.

\section{APPENDIX: NORMALIZATION OF LIGHT SPECTRA}

The number of photons per energy is governed by the number of tunneling electrons and their probability of undergoing a $|i\rangle \rightarrow|f\rangle$ decay. This probability, given by Fermi's golden rule [10], is enhanced at the frequency of cavity resonant modes in the surrounding of the emitter, the so-called Purcell's effect [31]. In the STM junction, the modes are those due to surface plasmon states confined in the cavity formed by the tip and the sample.

Surface plasmons of noble metals typically exhibit resonances in the window between $500 \mathrm{~nm}$ and $1000 \mathrm{~nm}$ spectral wavelength $[32,33]$. These plasmons dominate the spectral function of the emitted photons, appearing as features [as in Fig. 9(a)] that crucially depend on the tip shape [32-35].
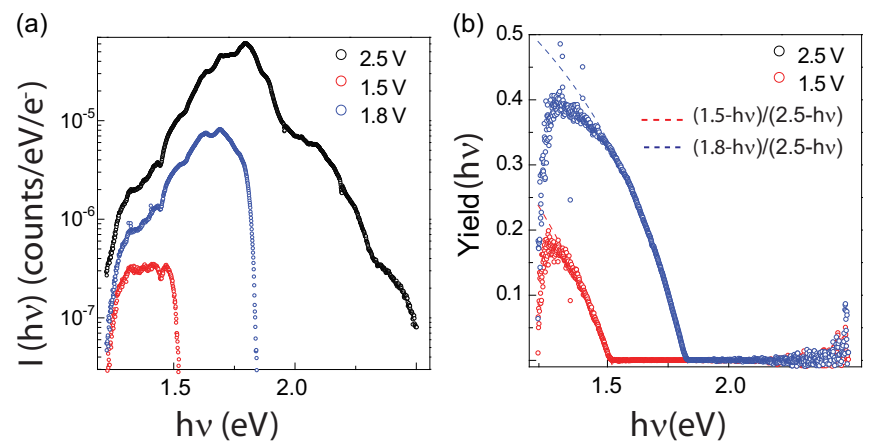

FIG. 9. (Color online) (a) Set of light emission spectra obtained on a bare $\operatorname{Ag}(111)$ surface using a W tip, presumably coated by silver after indentations into the substrate. Each spectrum is measured by recording photons for 4 minutes with the STM feedback stabilized at $I=5 \mathrm{nA}$ and at different sample bias values. The spectra are divided by the number of tunneling electrons $I_{t} \times t$. (b) Spectral yield obtained by dividing each of the spectra in (a) by the spectrum recorded at the highest bias value $(2.5 \mathrm{~V})$ and by an additional factor of 2.2 and 1.7 introduced to fit the resulting Yield with the dashed model function $\frac{|e V|-h v}{\left|e V_{b g}\right|-h v}$ described in the text. The origin of this constant factor is probably due to a decrease in the plasmon enhancement with tip-sample separation. 
To remove such tip-dependent plasmonic features, and to be able to qualitatively compare data from different tips and under different tunneling parameters, we normalize light emission spectra by the electron current and time (i.e., the number of electrons used for the experiment) and by a reference spectrum $I_{\text {ref }}(h v)$ taken with the corresponding tip on a bare metal region (also normalized by current and time). We name the resulting normalized spectra as "spectral yield" in the paper, defined as $Y(h v)=I(h v) / I_{\text {ref }}(h v)$. The reference spectrum is measured at a bias voltage $V_{\mathrm{bg}}$ lying well above the spectral range of the plasmonic features and, hence, of the photon emission energy range.

The number of photons emitted with a certain energy value $h v$ depends also on the possible number of $|i\rangle \rightarrow$ $|f\rangle$ transitions allowing such photon emission within the energy window defined by the applied bias. The number of such transitions thus scales as $\int_{h v}^{e V} d \varepsilon \sim|e V|-h v$, where necessarily $|e V|>h v$. Hence, there are fewer photons as their energy approaches $e V_{s}$. Such behavior persists when the spectra is normalized by a background spectrum taken at $V_{b g}>V$. In this case, the spectral yield function $Y(h v)$ decreases smoothly as $\propto \frac{|e V|-h v}{\left|e V_{b g}\right|-h v}$. Figure 9(b) shows that, as explained here, the normalized light spectra on a bare metal from Fig. 9(a) follow a monotonically decaying plot becoming zero at photon energies equal to $e V_{s}$. This is also the behavior of yield data in Fig. 6(b) of the main text.

The spectral yield as in Fig. 9(b) is a tip-independent function that can be used to compare data quantitatively from several tips (as far as each is normalized separately), with the particular care that the tips need to be active for emitting light in the energy range of inspection.
[1] K. T. Shimizu, W. K. Woo, B. R. Fisher, H. J. Eisler, and M. G. Bawendi, Phys. Rev. Lett. 89, 117401 (2002).

[2] M. Fu et al., Appl. Phys. Lett. 100, 063117 (2012).

[3] K. J. Savage et al., Nature (London) 491, 574 (2012).

[4] C. Navio, M. J. Capitán, J. Álvarez, F. Yndurain, and R. Miranda, Phys. Rev. B 76, 085105 (2007), and references therein.

[5] J. M. Burkstrand, G. G. Kleiman, G. G. Tibbetts, and J. C. Tracy, J. Vac. Sci. Technol. 13, 291 (1976).

[6] D. Heskett, A. Baddorf, and E. W. Plummer, Surf. Sci. 195, 94 (1988).

[7] D. M. Borsa and D. O. Boerma, Surf. Sci. 548, 95 (2004).

[8] D. Ecija, J. Gallego, and R. Miranda, Surf. Sci. 603, 2283 (2009).

[9] C. F. Hirjibehedin, C. P. Lutz, and A. J. Heinrich, Science 312, 1021 (2006).

[10] F. Rossel, M. Pivetta, and W. D. Schneider, Surf. Sci. Rep. 65, 129 (2010).

[11] R. Berndt, J. K. Gimzewski, and P. Johansson, Phys. Rev. Lett. 67, 3796 (1991).

[12] P. Bharadwaj, A. Bouhelier, and L. Novotny, Phys. Rev. Lett. 106, 226802 (2011).

[13] U. Hakanson, M. K.-J. Johansson, M. Holm, C. Pryor, L. Samuelson, W. Seifert, and M.-E. Pistol, Appl. Phys. Lett. 81, 4443 (2002).

[14] T. K. Johal, G. Pagliara, R. Rinaldi, A. Passaseo, R. Cingolani, M. Lomascolo, A. Taurino, M. Catalano, and R. Phaneuf, Phys. Rev. B 66, 155313 (2002).

[15] P. Myrach, N. Nilius, and H.-J. Freund, Phys. Rev. B 83, 035416 (2011).

[16] G. Hoffmann, J. Kliewer, and R. Berndt, Phys. Rev. Lett. 87, 176803 (2001).

[17] G. Nazin, X. Qiu, and W. Ho, Phys. Rev. Lett. 90, 216110 (2003).

[18] C. Chen, C. A. Bobisch, and W. Ho, Science 325, 981 (2009).

[19] X. H. Qui, G. V. Nazin, and W. Ho, Science 299, 542 (2003).

[20] E. Cavar, M.-C. Blüm, M. Pivetta, F. Patthey, M. Chergui, and W.-D. Schneider, Phys. Rev. Lett. 95, 196102 (2005).
[21] Z.-C. Dong, X.-L. Guo, A. Trifonov, P. Dorozhkin, K. Miki, K. Kimura, S. Yokoyama, and S. Mashiko, Phys. Rev. Lett. 92, 086801 (2004).

[22] C. Chen, P. Chu, C. A. Bobisch, D. L. Mills, and W. Ho, Phys. Rev. Lett. 105, 217402 (2010).

[23] F. J. Giessibl, Rev. Mod. Phys. 75, 949 (2003).

[24] X.-D. Ma, D. I. Bazhanov, O. Fruchart, F. Yildiz, T. Yokoyama, M. Przybylski, V. S. Stepanyuk, W. Hergert, and J. Kirschner, Phys. Rev. Lett. 102, 205503 (2009).

[25] H. Wu and W. Chen, J. Am. Chem. Soc. 133, 15236 (2011).

[26] A. Soon, L. Wong, B. Delley, and C. Stampfl, Phys. Rev. B 77, 125423 (2008).

[27] K. Bhattacharjee, X.-D. Ma, Y. Q. Zhang, M. Przybylski, and J. Kirschner, Surf. Sci. 606, 652 (2012).

[28] C. D. Ruggiero, M. Badal, T. Choi, D. Gohlke, D. Stroud, and J. A. Gupta, Phys. Rev. B 83, 245430 (2011).

[29] The fit of data shown in Fig. 8(a) is done using a combination of a Gaussian function on top of a linear background. The latter reproduces the light arising from electron decay into resonance $\mathrm{S}_{1}$, which is linear with $e V_{s}-h v$ (see the Appendix).

[30] The measurements are done in constant-current mode. The opening of a new resonant tunneling channel leads to a tip retraction by $\sim 1 \AA$ to maintain the current constant. The weakening of the inelastic emission from transitions to lower levels is a consequence of the larger fraction of resonant tunneling electrons in the current. Resonant electrons may emit at different wavelengths.

[31] E. M. Purcell, Phys. Rev. 69, 681 (1946).

[32] J. Aizpurua, S. P. Apell, and R. Berndt, Phys. Rev. B 62, 2065 (2000).

[33] Kazuyuki Meguro, Kenji Sakamoto, Ryuichi Arafune, Minoru Satoh, and Sukekatsu Ushioda, Phys. Rev. B 65, 165405 (2002).

[34] Ping Chu and D. L. Mills, Phys. Rev. B 84, 045430 (2011).

[35] R. W. Rendell and D. J. Scalapino, Phys. Rev. B 24, 3276 (1981).

[36] I. Horcas et al., Rev. Sci. Instrum. 78, 013705 (2007). 